\title{
Constrained Optimization of Monopulse Circular Aperture Distribution in the Presence of Blockage
}

\author{
M. SACHIDANANDA AND S. RAMAKRISHNA
}

\begin{abstract}
A method of synthesizing aperture antenna excitations to obtain optimum performance in the presence of aperture blockage is presented. The problem of optimizing the performance indices such as directivity and angular sensitivity is formulated as an $\mathrm{N}$-dimensional minimization problem, with constraint on the sidelobe level. In most cases, the objective function is nonlinear with multiple minima which does not yield readily to gradient methods. A simplex search method is used in the study for optimizing the performance index. The sidelobe level (SLL) constraint has been incorporated using the penalty function method. The method is applied to circular monopulse aperture distribution with circular blockage to obtain maximum directivity factor (DF) in the sum mode and maximum angular sensitivity factor (ASF) in the difference mode, with sidelobe level constraint. The effect of blockage on the maximum directivity and maximum angular sensitivity is studied for various sidelobe level constraints.
\end{abstract}

\section{INTRODUCTION}

$\mathbf{F}$ RONT-FED reflector antennas, in view of their simplicity, are widely used for communication and radar applications. One of the major problems encountered in the design and optimization of performance of these antennas is that of aperture blockage. This problem is more pronounced in aircraft and missile antennas, where the limitation on the size of the total aperture makes the blockage a significant proportion of the aperture. Because of the stringent requirements on these airborne antennas, it is necessary to obtain the best performance out of the available aperture size.

A number of synthesis techniques have been reported in the literature for optimizing the performance of aperture distribution in the absence of blockage. For example, Taylor [1] has studied circular aperture distributions that give optimum beamwidth for a given sidelobe level. He has defined an ideal pattern that gives the narrowest beamwidth and synthesized this pattern using Woodward's sampling synthesis. Ruze [2] has extended the circular aperture synthesis to arbitrary aperture distribution by expanding the aperture distribution in terms of properly orthogonalized Bessel functions to obtain the sampling property. Rhodes [3] has studied extensively the pattern space factors for rectangular and circular apertures. Several authors [4]-[6] have studied the analysis and synthesis of monopulse difference patterns. Bayliss [6] has used an ideal difference pattern similar to Taylor's equal sidelobe pattern with optimum properties, suitably modified to obtain the required sidelobe taper. Sanzgiri et al. [7] have reported an eigenvalue method to optimize the performance indices, such as directivity, beam efficiency, and angular sensitivity of the monopulse aperture distribution with constraint on the sidelobe level. All these methods are confined to regular aperture geometries, such as linear, circular and rectangular apertures, and cannot be easily applied for the case of blocked aperture. The

Manuscript received March 2, 1982; revised October 18, 1982.

The authors are with Department of Aeronautical Engineering, Indian Institute of Science, Bangalore 560 012, India. common methodology used by all these consists of first defining or constructing an aperture-limited pattern function with optimum properties desired, and then synthesizing this pattern using the available synthesis techniques. In the case of aperture shapes of regular geometries without blockage, the realizable pattern function has an important property, namely, uniformly decaying sidelobe structure. When blockage is introduced, this uniformity vanishes which makes the definition of optimum aperture limited pattern function very difficult.

Presented in this paper is a method for optimizing the performance indices of an aperture distribution that can incorporate the effect of aperture blockage. The performance index under consideration is optimized directly by formulating an objective function, consisting of performance index and the sidelobe level constraints, and using a numerical search algorithm for objective function minimization. The method is applied to a circular aperture with circular blockage, and aperture distributions are obtained that maximize the directivity factor (DF) in the sum mode and angular sensitivity factor (ASF) in the difference mode of monopulse antenna, with constraint on the sidelobe level (SLL).

\section{OPTIMIZATION IN THE PRESENCE OF BLOCKAGE}

Optimum design of an aperture distribution is one of determining the shape of the distribution that maximizes the performance index subject to certain constraints, imposed by the practical realizability and the specification requirements. Any arbitrary distribution $F(x)$ over an interval $\left(x_{1}, x_{2}\right)$ can be expanded in terms of a set of functions $f_{1}, f_{2}, \cdots, f_{\infty}$ which form an orthogonal and complete set in the interval $\left(x_{1}, x_{2}\right)$

$$
F(x)=\sum_{i=1}^{\infty} C_{i} f_{i}(x)
$$

where $x$ is the aperture variable and $C_{i}$ are the complex coefficients determining the amplitude and phase of each function vector. For real distribution $F(x)$, both $C_{i}$ and $f_{i}$ are real. The functions $f_{i}$ are called the basis functions which define the directions in the function space. Now the optimization problem can be stated as one of obtaining the coefficients $C_{i}$ that maximizes the performance index. The coefficients form the variables of optimization.

A finite set of functions $f_{i}, i=1,2, \cdots, N$, define a subspace of dimensions $N$. In practice there is a limitation on the realizable aperture distribution; hence, in order that the synthesized distribution be realizable, the search space in the function domain must be limited to realizable shapes. It is often difficult in antenna problems to define the realizable space, but the distribution shapes can be approximately confined to realizable shapes by proper choice of basis functions and number of terms $N$ to define a subspace.

Another point of importance to the method proposed is that 
the linear independence of basis function is a sufficient condition for the coefficients $C_{i}$ to be unique. And, if a set of functions $f_{i}$, $i=1,2, \cdots, N$, is orthogonal over an interval $\left(x_{1}, x_{2}\right)$ with a weight function $w(x)$, then, in the subinterval $\left(x_{1}, x_{3}\right)$, where $x_{1}<x_{3}<x_{2}$, the functions remain linearly independent.

The implication of this is that if a set of orthogonal functions is chosen for expanding the aperture distribution without blockage, the same set of functions can be used even when the blockage is introduced because the functions still remain linearly independent, giving a unique set of coefficients $C_{i}$.

As in the previous methods, the aperture distribution is expanded in terms of a set of basis function which define the search space in the function domain:

$$
F(x)=\sum_{i=1}^{N} C_{i} f_{i}(x)
$$

where $F(x)$ is the distribution function, $f_{i}(x), i=1,2, \cdots, N$ are the basis functions, and $C_{i}, i=1,2, \cdots, N$ are the coefficients. The linear independence of basis functions is a sufficient condition for the set of coefficients $C_{i}$ to be unique. The optimization problem is one of determining the value of the coefficients $C_{i}$ that maximizes the performance index subject to constraint on the sidelobe level. Coefficients $C_{i}$ form the variables of optimization.

The choice of basis functions $f_{i}$ depends on the aperture geometry and nature of the aperture distribution desired. For example, the aperture distribution over a circular aperture for a pencil beam pattern, is circularly symmetric with a taper in the radial direction. This type of distribution can be expanded in terms of properly orthogonalized zero-order Bessel functions in the radial directions which are mathematically easy to manipulate in the cylindrical coordinate system.

The method of optimizing the performance index of aperture distribution consists of formulating an objective function consisting of 1) the performance index, expressed in terms of the basis functions and the unknown coefficients, and 2) the sidelobe constraint function, formulated as the penalty function, also expressed in terms of the basis functions and unknown coefficients, and then minimizing this objective function. This objective function is nonlinear in unknown coefficients $C_{i}$ in most cases and has multiple minima. A simplex search method of Nelder and Mead [8] has been used to minimize the objective function.

\section{SIDELOBE LEVEL CONSTRAINT}

Because of the irregular nature of the sidelobe structure of the pattern of a blocked aperture, all the sidelobe peaks need not necessarily be equal for the optimum pattern. Hence, the sidelobe level constraint is specified as inequality constraint in the present study. The sidelobe level will be equal to the specified constraint level only at the peak of some of the sidelobes for the optimum pattern, and it is sufficient to constrain the sidelobe level at these peak locations. But for the numerical search method employed for optimization, this has been found to be unsatisfactory, since locating the peaks for every iteration is a time-consuming process. Certain general characteristics of the pattern of a blocked aperture with uniform distribution have been used to evolve a new procedure of controlling the sidelobe level. Though the pattern sidelobe structure is irregular for a blocked aperture, the decaying nature of sidelobe peaks, with increasing angular displacement from the main beam, is still present. For a given blockage, an angular sector immediately next to the main beam can be defined, in which the sidelobe level of the pattern of uniform distribution exceeds the specified constraint level. The sidelobe level is constrained at a number of closely spaced locations in this angular sector to keep the constraint violation within acceptable limits. This method is computationally faster because the major portion of the computation needs to be done only once in the beginning of the iterative process, and can then be stored in the computer memory.

If $u_{1}$ is the approximate first null of the pattern in $u=\sin \theta$ space of a uniform aperture distribution with blockage and $u_{M}$ is the maximum angle from main beam peak beyond which the sidelobe level is less than the constraint level " $e$," then the angular sector $u_{1}$ to $u_{M}$ is divided into $M$ regularly spaced points and the SLL is computed at each of the points. The constraint on SLL is applied in the form of a penalty function $P$ formulated as follows:

$$
P=p \cdot \sum_{i=1}^{M} \max \left(0, L\left(u_{i}\right)-e\right)
$$

where $p$ is the penalty value, $L\left(u_{i}\right)$ is the sidelobe level at $u_{i}$, and $e$ is the constraint level given as power ratio. The function $\max (\cdot)$ indicates that the greater of the two values in the brackets is taken as the value of the function. This ensures that locations $u_{i}$, where the SLL is higher than $e$, only contribute to penalty function.

The penalty function $P$ is added to the negative of the performance index to be maximized to form an objective function. In the process of minimization, the penalty value $p$ is set to a small value to start with and is increased in steps until the objective function value remains stationary. The objective function value becomes independent of penalty value $p$ when the sidelobe constraint is satisfied.

An advantage to this method of applying the SLL constraint is that it can be used to contain the sidelobes in an envelope by specifying different constraint levels " $e_{i}$ " at each $u_{i}$ to form a constraint envelope.

The effectiveness of the method lies in the formulation of the objective function which is a nonlinear function of the unknown coefficients $C_{i}$ which form the variables of optimization. The objective function is obtained in a form where all the known parts involving the basis function, $f_{i}$ are precomputed and the values stored in the computer memory. This makes the objective function computation for every iteration very simple and fast.

The numerical aspects of minimization will be discussed in the following application to optimization of monopulse distribution.

\section{OPTIMIZATION OF CIRCULAR MONOPULSE APERTURE DISTRIBUTION}

\section{A. Aperture Distribution Expansion and Choice of Basis Functions}

A circular aperture of radius $a$ in the $(x, y)$ plane with the center of the aperture coinciding with the origin is considered. $Z$-axis is the main beam direction or the boresight axis. A circular area of radius $b$ is assumed to be blocked and completely absorbing. The ratio $b / a$ is termed blockage.

Expansion of any arbitrary aperture distribution over a circular aperture in terms of properly orthogonalized Bessel functions has been given by Ruze [2]. The Bessel functions form a natural basis in the cylindrical geometry used, and are easy to manipulate mathematically.

The sum and difference mode distributions for a monopulse 
circular aperture can be expanded in terms of zero-order and first-order Bessel functions, respectively, because of the even and odd nature of the distribution.

$$
\begin{aligned}
& F_{\Sigma}\left(\rho, \varphi^{\prime}\right)=\sum_{m=1}^{N} C_{m 0} J_{0}\left(\alpha_{m 1} \frac{\rho}{a}\right) \\
& F_{\Delta}\left(\rho, \varphi^{\prime}\right)=\sum_{m=1}^{N} C_{m 1} J_{1}\left(\alpha_{m 0} \frac{\rho}{a}\right) \cos \varphi^{\prime}
\end{aligned}
$$

where $F_{\Sigma}$ and $F_{\Delta}$ are $N$ terms approximation to the sum mode and difference mode aperture amplitude distributions, $J_{0}$ and $J_{1}$ are the Bessel functions of zero and first order, $\rho$ and $\varphi^{\prime}$ are the cylindrical polar coordinates in the aperture plane. The phase distribution over the aperture is assumed to be uniform making the unknown coefficients $C_{m o}$ and $C_{m 1}$ real. The normalizing constants $\alpha_{m 0}$ and $\alpha_{m 1}$ are the roots of Bessel function, $\alpha_{m n}$ representing the $m$ th root of $n$th order Bessel function, to make the Bessel function orthogonal over the interval $(0, a)$ of the aperture. Taylor [1] has shown that the lowest far sidelobe level decay rate occurs for nonzero edge illumination of the aperture and has better control on the sidelobe level for a given number of terms in the aperture distribution expansion [6]. Hence the normalizing constants $\alpha_{m 0}$ and $\alpha_{m 1}$ are chosen to give a pedestal at the edge.

\section{B. Derivation of Expressions for Pattern and Radiated Power in the Sum and Difference Modes}

The expressions for the radiation patterns for the sum and difference mode distributions can be obtained by taking the Fourier transform of the aperture distributions. The sum and difference mode patterns, $g_{\Sigma}$ and $g_{\Delta}$ can be written as

$$
g_{\Sigma}(u, \varphi)=\int_{0}^{2 \pi} \int_{b / a}^{1} F_{\Sigma}\left(r, \varphi^{\prime}\right) e^{j k} 0^{u a r \cos \left(\varphi-\varphi^{\prime}\right)} r d r d \varphi^{\prime}
$$

and

$$
g_{\Delta}(u, \varphi)=\int_{0}^{2 \pi} \int_{b / a}^{1} F_{\Delta}\left(r, \varphi^{\prime}\right) e^{j k} 0^{a u r \cos \left(\varphi-\varphi^{\prime}\right)} r d r d \varphi^{\prime}
$$

where $u=\sin \theta$ and $\varphi$ are pattern variables in the spherical coordinate system, $k_{0}$ is the free space propagation constant, and $r=\rho / a$, is the normalized aperture variable. The integration is confined to aperture surface only because the field is assumed to be zero outside the aperture and in the blocked region. After integrating and substituting the limits the pattern expression can be obtained in the form

$$
g_{\Sigma}(u)=\sum_{m=1}^{N} C_{m 0} I_{m}^{0}(u)
$$

and

$$
g_{\Delta}(u)=\sum_{m=1}^{N} C_{m 1} I_{m}^{1}(u, \varphi)
$$

where

$$
\begin{aligned}
& I_{m}^{0}(u) \\
& =\frac{2 \pi a^{2}}{\alpha_{m 1}^{2}-\left(k_{0} a u\right)^{2}}\left[-k_{0} a u J_{0}\left(\alpha_{m 1}\right) J_{1}\left(k_{0} a u\right)\right. \\
& \quad-\frac{b}{a} \alpha_{m 1} J_{1}\left(\alpha_{m 1} \frac{b}{a}\right) J_{0}\left(k_{0} u b\right) \\
& \left.\quad+k_{0} u b J_{0}\left(\alpha_{m 1} \frac{b}{a}\right) J_{0}\left(k_{0} u b\right)\right], \text { for } k_{0} a u \neq \alpha_{m 1}
\end{aligned}
$$

$$
\begin{aligned}
& I_{m}^{0}(u) \\
& =\pi a^{2}\left[J_{0}^{2}\left(\alpha_{m 1}\right)-\left(\frac{b}{a}\right)^{2}\left\{J_{0}^{2}\left(\alpha_{m 1} \frac{b}{a}\right)+J_{1}^{2}\left(\alpha_{m 1} \frac{b}{a}\right)\right\}\right] \\
& I_{m}^{1}(u, \varphi) \\
& =\frac{j \pi a^{2} \cos \varphi}{\alpha_{m 0}^{2}-\left(k_{0} a u\right)^{2}}\left[\alpha_{m 0} J_{2}\left(\alpha_{m 0}\right) J_{1}\left(k_{0} a u\right)-\alpha_{0} a u J_{1}\left(\alpha_{m 0}\right)\right. \\
& \quad \cdot J_{2}\left(k_{0} a u\right)-\alpha_{m 0} \frac{b}{a} J_{2}\left(\alpha_{m 0} \frac{b}{a}\right) J_{1}\left(k_{0} u b\right)+k_{0} u b \\
& \left.\quad \cdot J_{1}\left(\alpha_{m 0} \frac{b}{a}\right) J_{2}\left(k_{0} u b\right)\right] \quad \text { for } k_{0} a u \neq \alpha_{m 0} \\
& I_{m}^{1}(u, \varphi) \\
& =\frac{j \pi a^{2} \cos \varphi}{2}\left[J_{1}^{2}\left(\alpha_{m 0}\right)-\left(\frac{b}{a}\right)^{2}\left\{J_{1}^{2}\left(\alpha_{m 0} \frac{b}{a}\right)\right.\right. \\
& \left.\left.\quad-J_{2}\left(\alpha_{m 0} \frac{b}{a}\right) \cdot J_{0}\left(\alpha_{m 0} \frac{b}{a}\right)\right\}\right], \quad \text { for } k_{0} a u=\alpha_{m 0} \cdot(13)
\end{aligned}
$$

The radiated power from the aperture can be computed using the Poynting vector theorem [9]. The expressions for the sum and difference mode radiated powers can be obtained in the form

$$
\begin{aligned}
& P_{r \Sigma}=\sum_{m=1}^{N} \sum_{n=1}^{N} C_{m 0} C_{n 0} Q_{m n}^{0} \\
& P_{r \Delta}=\sum_{m=1}^{N} \sum_{n=1}^{N} C_{m 1} C_{n 1} Q_{m n}^{1}
\end{aligned}
$$

where $P_{r \Sigma}$ and $P_{r \Delta}$ are the radiated power in the sum and difference modes. The expressions $Q_{m n}^{0}$ and $Q_{m n}^{1}$ are given by

$$
\begin{aligned}
Q_{m n}^{0}= & \frac{2 \pi a^{2}}{\alpha_{m 1}^{2}-\alpha_{n 1}^{2}}\left[-\alpha_{m 1} J_{1}\left(\alpha_{m 1} \frac{b}{a}\right) J_{0}\left(\alpha_{n 1} \frac{b}{a}\right)\right. \\
& \left.+\alpha_{n 1} J_{0}\left(\alpha_{m 1} \frac{b}{a}\right) J_{1}\left(\alpha_{n 1} \frac{b}{a}\right)\right], \quad m \neq n \\
Q_{m n}^{0}= & \pi a^{2}\left[J_{0}^{2}\left(\alpha_{m 1}\right)-\left(\frac{b}{a}\right)^{2}\left\{J_{0}^{2}\left(\alpha_{m 1} \frac{b}{a}\right)\right.\right. \\
& \left.\left.+J_{1}^{2}\left(\alpha_{m 1} \frac{b}{a}\right)\right\}\right], \quad m=n
\end{aligned}
$$

$$
\begin{aligned}
Q_{m n}^{1}= & \frac{\pi a b}{\alpha_{m 0}^{2}-\alpha_{n 0}^{2}}\left[\alpha_{m 0} J_{2}\left(\alpha_{m 0}\right) J_{1}\left(\alpha_{n 0}\right)-\alpha_{n 0} J_{1}\left(\alpha_{m 0}\right)\right. \\
& \cdot J_{2}\left(\alpha_{n 0}\right)-\left(\frac{b}{a}\right)\left\{\alpha_{m 0} J_{2}\left(\alpha_{m 0} \frac{b}{a}\right) J_{1}\left(\alpha_{n 0} \frac{b}{a}\right)\right. \\
& \left.\left.-\alpha_{n 0} J_{1}\left(\alpha_{m 0} \frac{b}{a}\right) J_{2}\left(\alpha_{n 0} \frac{b}{a}\right)\right\}\right], \quad m \neq n \\
Q_{m n}^{1}= & \frac{\pi a^{2}}{2}\left[J_{1}^{2}\left(\alpha_{m 0}\right)-\left(\frac{b}{a}\right)^{2}\left\{J_{1}^{2}\left(\alpha_{m 0} \frac{b}{a}\right)-J_{0}\left(\alpha_{m 0} \frac{b}{a}\right)\right.\right. \\
& \left.\left.\cdot J_{2}\left(\alpha_{m 0} \frac{b}{a}\right)\right\}\right], \quad m=n
\end{aligned}
$$




\section{Objective Function for Sum-Mode DF Optimization}

The directivity of an antenna in the direction $(u, \varphi)$ is defined as the ratio of the radiation intensity in the direction $(u, \varphi)$ to the average radiation intensity. The directivity of the circular aperture in the sum mode and in the boresight direction $(0,0)$ can be expressed as

$$
D(0)=\frac{4 \pi}{\lambda^{2}} \frac{\left|g_{\Sigma}(0)\right|^{2}}{P_{r \Sigma}}
$$

where $D(0)$ is the directivity in the direction of boresight and $\lambda$ is the free space wavelength.

Normalizing this directivity with respect to the maximum directivity of the antenna with uniform phase and amplitude distribution and zero blockage, the expression for the directivity factor (DF), which is also sometimes termed aperture efficiency, is obtained. The maximum directivity of a circular aperture of radius $a$ is given by

$$
D_{0}=\frac{4 \pi a^{2}}{\lambda^{2}}
$$

Hence, DF can be expressed as

$$
\frac{D(0)}{D_{0}}=\frac{\left|g_{\Sigma}(0)\right|^{2}}{\pi a^{2} P_{r \Sigma}}
$$

The SLL in the direction $u$ is defined as the ratio of the power in the direction $u$ to the power in the main beam peak direction and is expressed as

$$
L(u)=\left|\frac{g(u)}{g(0)}\right|^{2}
$$

The penalty function $P$ is formulated as explained in Section III by applying the constraint at $M$ points in the angular region $u_{1}$ to $u_{m}$ of $u$ space, at regular intervals.

$$
P=p \sum_{i=1}^{M} \max \left(0 ; \quad L\left(u_{i}\right)-e\right)
$$

The number of constraint locations $M$ is chosen depending on the accuracy to which the SLL has to be controlled to the specified level $e$. Since the locations $u_{i}$ are fixed, a sidelobe peak can fall in between two successive locations $u_{i}$ and $u_{i+1}$, and hence the peak level can be greater than $e$. This positive excursion can be kept small by making the spacings between successive locations small. Normally four to five locations per standard beamwidth $(u$ in standard BW is defined as $u=2 a / \lambda \sin \theta$ standard $\mathrm{BW}$ ) are found to be sufficient to limit this positive excursion to less than 0.5 $\mathrm{dB}$, which generally occurs only at the first one or two sidelobe peaks.

The objective function for DF optimization is thus formulated by adding the penalty function to the negative of the DF.

$$
\begin{aligned}
\phi_{0}= & -\frac{D(0)}{D_{0}}+P=-\frac{\sum_{m=1}^{N} C_{m 0} I_{m}^{0}(0)}{\pi a^{2} \sum_{m=1}^{N} \sum_{n=1}^{N} C_{m 0} C_{n 0} Q_{m n}^{0}} \\
& +p \cdot \sum_{i=1}^{N} \max \left(0 ; \frac{\sum_{m=1}^{N}\left(C_{m 0} I_{m}^{0}\left(u_{i}\right)\right.}{\sum_{m=1}^{N} C_{m 0} I_{m}^{0}(0)}-e\right) .
\end{aligned}
$$

This is a nonlinear function in the unknown coefficients $C_{m 0}$ : which are used as the variables of optimization. The values of the expressions $I_{m}^{0}(0), I_{m}^{0}\left(u_{i}\right)$ and $Q_{m n}^{0}$ are computed in the beginning of the iterative process of minimization, and stored in the computer memory which makes the computation of the value of the $\phi_{0}$ for every iteration simple and fast.

\section{Objective Function Formulation for Difference Mode ASF Optimization}

The angular sensitivity of the difference mode pattern is defined as the slope at the boresight of the difference voltage pattern normalized with respect to the radiated power. The angular sensitivity at boresight is expressed as [6]

$$
K=\frac{\partial}{\partial u}\left[\frac{4 \pi}{\lambda^{2}} \frac{\left|g_{\Delta}(0,0)\right|^{2}}{P_{r \Delta}}\right]^{1 / 2} \frac{2 a}{\lambda} \text { volt } / \text { volt } / \text { standard BW }
$$

It has been shown that maximum angular sensitivity occurs for a linear odd distribution with zero blockage [10]. We denote this as $K_{0}$ which, for a circular aperture, is given by

$$
K_{0}=\frac{\pi a^{2}}{\lambda} \text { volt } / \text { volt } / \text { standard BW. }
$$

A relative measure of angular sensitivity is obtained by normalizing it with respect to $K_{0}$ and is termed angular sensitivity factor (ASF). Substituting the expressions for $g_{\Delta}$ in (26) and differentiating, the expression for ASF is obtained as

$$
\frac{K}{K_{0}}=\left[P_{r \Delta}\right]^{1 / 2} \sum_{m=1}^{N} C_{m 1} S_{m}
$$

where

$$
S_{m}=\frac{\alpha \pi^{1 / 2}}{\alpha_{m 0}}\left[J_{2}\left(\alpha_{m 0}\right)-\left(\frac{b}{a}\right)^{2} J_{2}\left(\alpha_{m 0} \frac{b}{a}\right)\right] .
$$

The penalty function is formulated as in the previous case, and is added to the negative of the ASF to form the objective function.

$$
\begin{aligned}
\phi_{1}= & -\frac{K}{K_{0}}+P=-\left[\sum_{m=1}^{N} \sum_{n=1}^{N} C_{m 1} C_{n 1} Q_{m n}^{1}\right]^{1 / 2} \\
& \cdot \sum_{m=1}^{N} C_{m 1} S_{m}+p \sum_{i=1}^{M} \max \left(0 ;\left|\frac{\sum_{m=1}^{N} C_{m 1} I_{m}^{1}\left(u_{i}, 0\right)}{\sum_{m=1}^{N} C_{m 1} I_{m}^{1}(0,0)}\right|^{2}-e\right) .
\end{aligned}
$$

Here the constraint locations are chosen to lie in the angular region $u_{1}$ to $u_{M}$ in the $\varphi=0$ plane, since the azimuth difference pattern is assumed to have an odd symmetry about $\varphi=\pi / 2$ plane.

\section{E. Computational Aspects of Minimization}

If $N$ terms are taken in the aperture distribution expansion, the $N$ coefficients $C_{m 0}, m=1,2 \cdots N$ or $C_{m 1}, m=1,2 \cdots N$ form a set of variables of optimization, and defines an $N$-dimensional space. A set of coefficients is termed a point in the $N$-dimensional space. A point where the value of the penalty function $P$ is zero is termed a feasible point. At such a point, the SLL is less than or equal to the constraint level at all the $M$ locations $u_{i}, i=1,2, \cdots, M$. A region of the $N$ dimensional search space containing only feasible points is called feasible region. 
The objective functions formulated for DF and ASF optimization are nonlinear in the unknown coefficients and can have multiple minima. Such functions do not easily yield to gradient methods because, these methods generally give the minimum nearest to the starting point. Hence, the success of the gradient methods depends on locating a starting point near the global minimum which is often difficult in the case of nonlinear functions.

The simplex search method of Nelder and Mead [8] which is a powerful method in handling nonlinear functions, has been used in this study. This has multiple starting points and has greater probability of locating the global minimum. For an $N$-dimensional function minimization the simplex method starts with a set of $(N+1)$ points, termed a simplex, the next iterative point being obtained by reflecting the point with the highest objective function value about the centroid of the rest of the $N$ points. The line joining the centroid and the point with the highest function value gives the general direction in which the function decreases and the simplex is expanded or contracted in this direction depending on the function value at the new point. The search is terminated when the difference between the highest and the lowest function values, among the points of the simplex, is less than a prespecified value.

It may be noted that the penalty function contributes to the objective function only in the nonfeasible region. The penalty value $p$ controls the steepness of the function in the nonfeasible region. If the starting simplex is far removed from the global minimum, a large penalty value can cause the simplex to settle down in a local minimum. To avoid this, the minimization is carried out with a small penalty value to start with and is decreased tenfold for every cycle of minimization with last minimum point as the starting point. The minimization is terminated when the minima obtained in the two successive cycles remains constant within a certain specified error. This process has been successfully used in the study.

Generally, the first cycle takes the maximum number of iterations to reach a minimum, the successive cycles of minimizations are faster, the starting point being near the minimum. This also eliminates the need for chosing the best penalty value, it being arrived at automatically.

The starting simplex, in most of the cases investigated, is formed around uniform distribution for the sum mode DF optimization, and odd distribution, given by the first term of (5), for the difference mode. The simplex is formed around this point by symmetrically perturbing each component by a fixed amount to give a new point. This starting point has been found to be satisfactory in most of the cases investigated. But for large blockages $(b / a>0.2)$ and very low SLL constraint the above starting point was found to give local minimum sometimes. These local minima are identified by their low DF value and also by the discontinuous value of the DF versus blockage curve for a given SLL constraint which otherwise is smooth. The failure of the method to locate global minimum in such cases is believed to be because of the steep variations in the objective function behavior.

Two alternative methods of starting the simplex search has been tried and found to be successful. First one is to optimize DF or ASF for a given blockage $b / a$ with successively lower SLL constraints, and using the previous minimum point as the starting point for the next lower SLL constraints. The second one uses random search method to locate a best starting point. In this method, a random number generating routine, available in the system library, is used to generate random points. The routine is called $N$ times to generate an $N$-dimensional point. A large number of points are generated using this process and the objective function value is computed at every point, and the point with the lowest function value is used as the starting point for the simplex routine. Normally $N^{2}$ or more points are needed to obtain a reasonably good starting point near the global minimum. It is expected that because of random distribution of the generated points in the entire search space, at least one point will fall near the global minimum.

\section{RESULTS AND DISCUSSION}

\section{A. Sum-Mode DF Optimization Results}

The objective function (25) was formulated with ten terms $(N=10)$ in the aperture distribution expansion (4), chosen on the basis of the results (Fig. 4) obtained. It can be seen from the figure that the improvement in the optimum $D F$ is marginal for $N$ greater than 10 . The objective function formulated to optimize DF of circular aperture with circular blockage is minimized to an accuracy of 0.0001 , for various blockages and SLL constraints.

The minimization program is run on IBM 360/44. The number of iterations to reach the minimum and hence the total iteration time is found to depend on constraint level and blockage, the time increasing with increasing blockage and lower sidelobe constraint level. For example, with a SLL constraint of $-25 \mathrm{~dB}$, the iteration time was $29 \mathrm{~s}$ with a total of 348 iterations for zero blockage. The iteration time was $87 \mathrm{~s}$ with a total of 1079 iterations, when the blockage 0.2 is introduced for the same SLL constraint. The number of iterations also depends on the number variables which is same as $N$, the number of terms taken in the aperture distribution expansion.

The optimum DF obtained for various blockages and SLL constraint has been presented in Table I along with that obtained by Sanzgiri et al. [7] for zero blockage, for comparison. These results are for line source whereas the one obtained by the pro. posed method are for circular aperture. Even so, they can be compared because the DF represents a relative measure and is a function of aperture distribution shape only in one dimension, the distribution being assumed to be uniform in the $\varphi^{\prime}$ direction in the case of circular aperture. The number of terms $N$ in the present method is equal to 10 in each case, whereas, they are different for each SLL constraint in Sanzgiri's results.

The results compare favorably when due consideration is given to the number of terms taken in each case. The number $t$ represents the number of sidelobes of equal height immediately next to main beam. This number is chosen in the eigenvalue method of Sanzgiri while defining the optimum pattern, whereas the number of equal lobes for the optimum pattern for the given set of parameters is automatically chosen in the present method. The number of sidelobes $t$ of equal height are also seen to be different, contributing to the difference in the DF value obtained in the two methods. The optimum DF falls sharply with increasing blockage for lower SLL constraints (Fig. 1). It can be seen that for a given blockage there is a lower limit for the realizable SLL, unlike in the unblocked case.

For an unblocked aperture, minimum beamwidth and maximum directivity solutions coincide only for low SLL constraints, where a large number of sidelobes adjacent to the main lobe obey equality constraint, but deviate for higher sidelobes. Whenever a SLL is pushed down or pulled up, from the pattern of uniform 


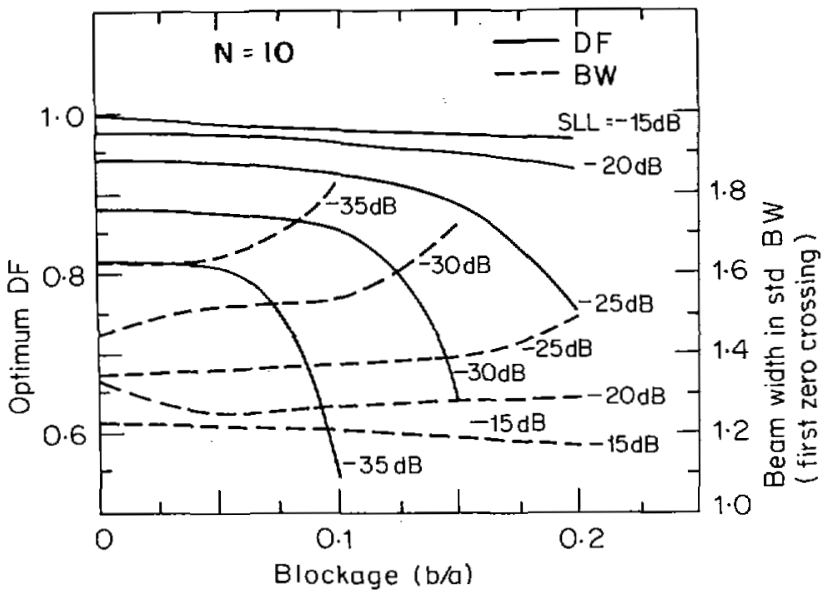

Fig. 1. Variation of optimum DF with blockage.

TABLE I

\begin{tabular}{lllllllllllll}
\hline \multicolumn{3}{c}{ Circular Aperture } & & & & & & $\begin{array}{c}\text { Line Source } \\
\text { Sanzgiti } \text { et al. [7] }\end{array}$ \\
\hline $\begin{array}{l}\text { Blockage } \\
b / a\end{array}$ & & 0.0 & & 0.1 & & 0.2 & & 0.0 & \\
\hline SLL & $N$ & $t$ & DF & $N$ & DF & $N$ & DF & $N$ & $t$ & DF \\
\hline$-15 \mathrm{~dB}$ & 10 & 1 & 1.000 & 10 & 0.979 & 10 & 0.962 & - & - & - \\
$-20 \mathrm{~dB}$ & 10 & 1 & 0.979 & 10 & 0.964 & 10 & 0.925 & 4 & 2 & 0.969 \\
$-25 \mathrm{~dB}$ & 10 & 2 & 0.941 & 10 & 0.922 & 10 & 0.748 & 9 & 6 & 0.927 \\
$-30 \mathrm{~dB}$ & 10 & 4 & 0.879 & 10 & 0.854 & 10 & - & 15 & 10 & 0.879 \\
$-35 \mathrm{~dB}$ & 10 & 6 & 0.819 & 10 & 0.543 & 10 & - & 23 & 18 & 0.833 \\
\hline
\end{tabular}

$t$ is the number of sidelobe peaks constrained to the specified level.

$N$ is the number of terms in the expansion of aperture distribution.

distribution, to satisfy an equality constraint, the BW increases or decreases, respectively, but the directivity always falls for both changes. The near sidelobes are affected more than the far sidelobes. Because of this differential effect on the BW, as the blockage is increased, the beam narrowing effect predominates due to the increase in the sidelobe level. Hence a decrease in the BW can be observed in Fig. 1, (first zero crossing of the pattern has been plotted) for SLL constraints $-15 \mathrm{~dB}$ and $-20 \mathrm{~dB}$. For lower SLL constraints the beam broadening effect predominates.

The aperture distributions $F_{\Sigma}\left(r, \varphi^{\prime}\right)$ obtained for optimum DF for the case of $b / a=0$ and $b / a=0.2$ has been presented in Fig. 2. The distributions synthesized are seen to be somewhat undulating in nature for lower SLL constraint and are difficult to realize in practice with small feeds. The undulating nature of the distribution is because no attempt has been made to constrain it to realizable shapes. Smoother distributions can be obtained by taking less number terms in the aperture distribution expansion, with some sacrifice in the DF. The variation of optimum distribution with number of terms is shown in Fig. 3. Fig. 4 shows the dependence of optimum DF on number of terms for various blockages. It can be seen that more number of terms are necessary with increasing blockage to avoid rapid fall of optimum DF.

The optimum patterns $g_{\Sigma}(u, \varphi)$ plotted inFig. 5 shows the irregular behavior in the sidelobe region when blockage is introduced. The pattern is plotted for a $10 \lambda$ aperture diameter. For $-25 \mathrm{~dB}$ SLL constraint, the constraint locations $u_{1}$ to $u_{M}$ are chosen to cover entire visible sidelobe region in the case of $b / a=$

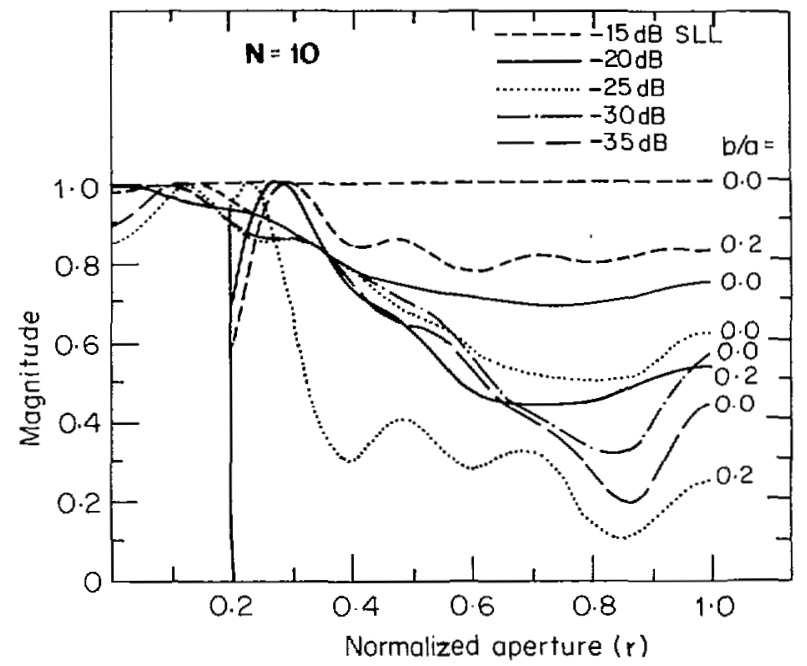

Fig. 2. Optimum sum-mode aperture distribution.

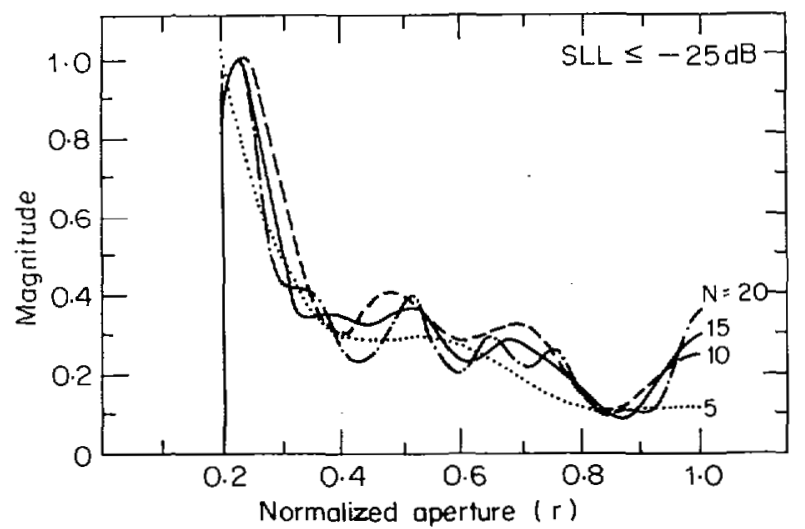

Fig. 3. Variation of optimum sum-mode aperture distribution with $N$.

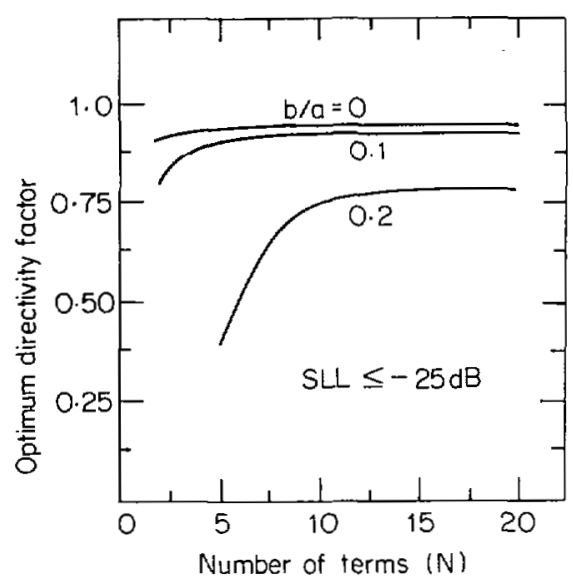

Fig. 4. Variation of optimum DF with $N$. 


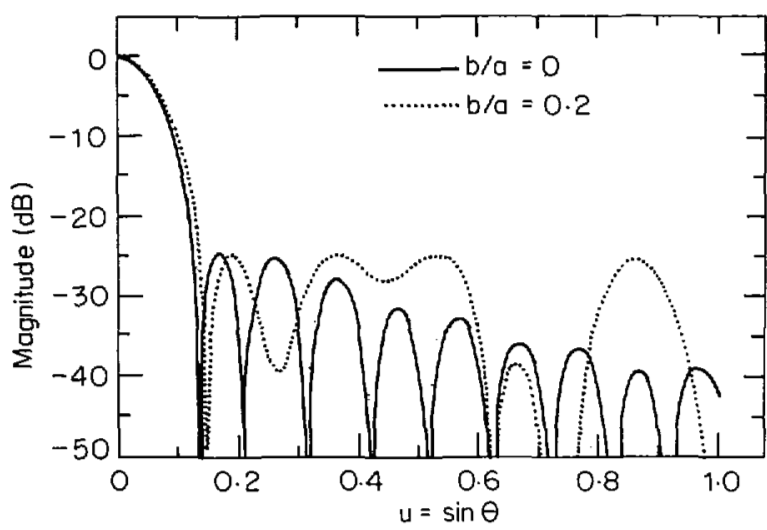

Fig. 5. Optimum sum-mode pattern.

TABLE II

CIRCULAR APERTURE ASF IN $d B=20$ LOG $_{10}\left[K(0) / K_{0}\right]$

Sanzgiri et al. [7]

\begin{tabular}{lccccccccc}
\hline $\begin{array}{l}\text { Blockage } \\
b / a\end{array}$ & & \multicolumn{1}{c}{0.0} & 0.1 & & 0.2 & \multicolumn{2}{c}{0.0} \\
\hline SLL & $N$ & ASF (dB) & $N$ & ASF (dB) & $N$ & ASF (dB) & $t$ & $N$ & ASF (dB) \\
\hline$-15 \mathrm{~dB}$ & 10 & -0.0945 & 10 & -0.0845 & 10 & -0.1185 & 2 & 5 & -0.1108 \\
$-20 \mathrm{~dB}$ & 10 & -0.3787 & 10 & -0.3825 & 10 & -0.4220 & 3 & 6 & -0.3696 \\
$-25 \mathrm{~dB}$ & 10 & -0.8059 & 10 & -0.8165 & 10 & -0.8980 & 5 & 10 & -0.8063 \\
$-30 \mathrm{~dB}$ & 10 & -1.3974 & 10 & -1.3958 & 10 & -2.2562 & 10 & 14 & -1.3514 \\
$-35 \mathrm{~dB}$ & 10 & -2.0328 & 10 & -2.1479 & 10 & - & - & - & -
\end{tabular}

$t$ is the number of sidelobe peaks constrained to the specified level.

$N$ is the number of terms in the expansion of aperture distribution.

0.2 since the sidelobe level rises to the constraint level right up to $u=0.9$, whereas for $b / a=0$, constraint need be applied in the region of first two sidelobes only.

\section{B. Optimization of Difference Mode ASF}

The optimum ASF optained for the circular aperture with $N=10$ is presented in Table II along with the results obtained by Sanzgiri et al. [7] for $b / a=0$. Here also the results are seen to compare well for $-25 \mathrm{~dB}$ SLL where the number of terms taken in both cases are equal. The difference in the two sets of results for other SLL constraints can be attributed to the difference in number of terms taken. ASF is expressed in terms of $\mathrm{dB}$ in all the cases. The effect of blockage can be seen to be much less on the difference pattern ASF because of the nature of distribution in the blocked region. The effect is more apparent when the SLL constraint is low.

Synthesized optimum distributions for $b / a=0.0$ and $b / a=$ 0.2 are given in Figs. 6 and 7, respectively. Here also the undulating nature of the distribution can be reduced by taking fewer terms in (5). The effect of blockage on the optinum ASF as well as on the corresponding beamwidth is less pronounced than on sum mode DF (Fig. 8). This is also reflected in the pattern sidelobe structure. The patterns for two cases, $b / a=0.0$ and 0.2 and $-30 \mathrm{~dB}$ SLL constraint is presented in Fig. 9 for comparison.

\section{CONCLUSION}

The method of optimizing the performance indices of aperture antenna presented in this paper is a direct approach and is most

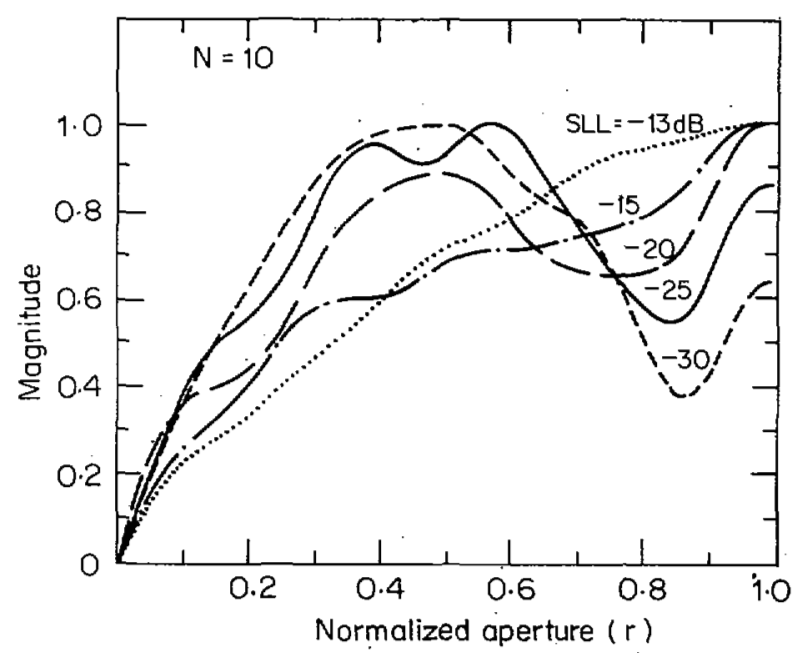

Fig. 6. Optimum difference mode aperture distribution, $b / a=0.0$.

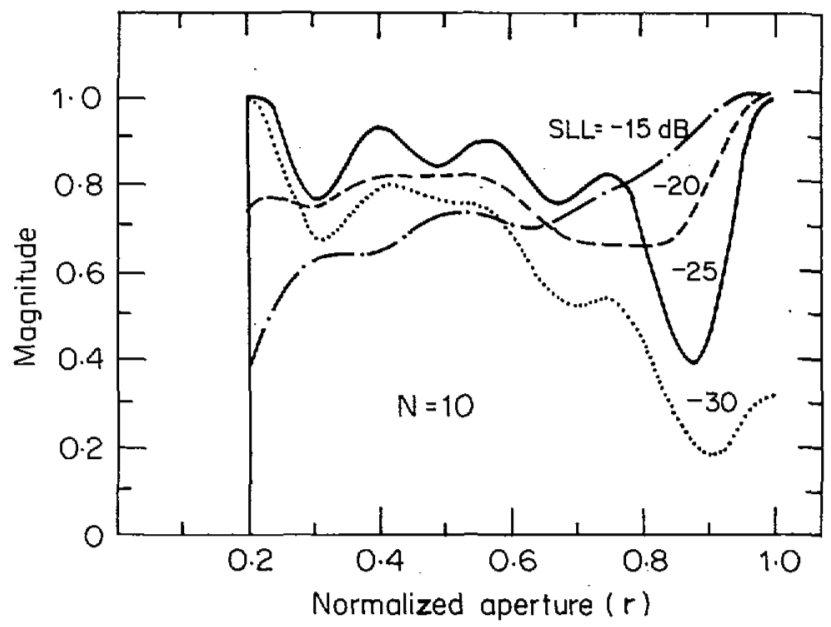

Fig. 7. Optimum difference mode aperture distribution, $b / a=0.2$.

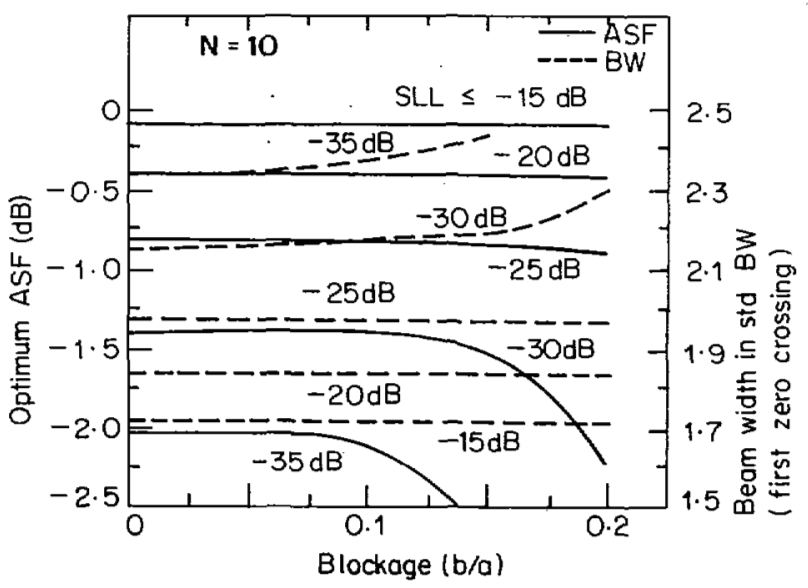

Fig. 8. Variation of optimum ASF with blockage. 


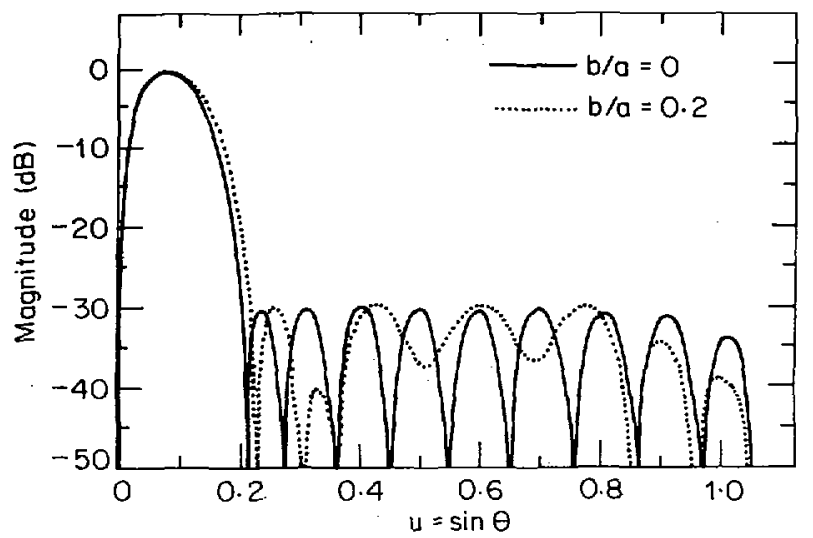

Fig. 9. Optimum difference mode pattern.

general in nature, enabling the method to be applied in a variety of aperture shapes, blockage shapes, and performance indices. The sidelobe constraint may also be applied as an envelope which includes the possibility of placement of null in a particular direction to minimize the interference signal.

\section{ACKNOWLEDGMENT}

The authors wish to thank Dr. P. R. Mahapatra for his valuable assistance in programming the simplex algorithm and fruitful discussions.

\section{REFERENCES}

[1] T. T. Taylor, "Design of circular apertures for narrow beam width and low sidelobes," IRE Trans. Antennas Propagat., vol: AP-8, pp. 1722, Jan. 1960.

[2] J. Ruze, "Circular aperture synthesis," IEEE Trans. Antennas Propagat., vol. AP-12, pp. 691-694, Nov. 1964.
[3] D. R. Rhodes, "On the aperture pattern space factors for rectangular and circular apertures," IEEE Trans. Antennas Propagat., vol. AP-19, pp. 763-770, Nov. 1971

[4] O. R. Price and R. F. Hyneman, "Distribution functions for monopulse antenna difference patterns," IRE Trans. Antennas Propagat., vol. AP.8, pp. 567-576, Nov. 1960.

[5] E. J. Powers, "Utilization of Lamda functions in the analysis and synthesis of monopulse antenna difference patterns," IEEE Trans. Antennas Propagat., vol. AP-15, pp. 771-777, Nov. 1967.

[6] E. T. Bayliss, "Design of monopulse antenna difference pattems with low side lobes," Bell Syst. Tech. J., pp. 623-650, May-June; 1968.

[7] S. M. Sanzgiri, J. K. Butler, and R. 'C. Voges, "Optimum aperture monopulse excitations," IEEE Trans. Antennas Propagat., vol. AP. 20, pp. 275-281, May 1972.

[8] J. A. Nelder and R. Mead, "Simplex method for function minimisation," Comput. J., vol. 7, pp. 308-313, 1965.

[9] S. Silver, Microwave Antenna Theory and Design. New York: McGraw-Hill, 1949, p. 162 (M.I.T. Radiation Lab. Series vol. 12).

[10] G. K. Kirkpatrick, "Final engineering report on angular accuracy improvement," Gen. Elec. Co. Rep., 1952.

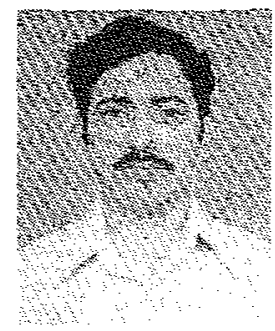

M. Sachidananda received the B.E. degree in electrical engineering from the Karnataka Regional Engineering College, Surathkal, India, in 1970 and the Ph.D. degree in antennas from the Indian Institute of Science, Bangalore, India, in 1980.

$\mathrm{He}$ joined the Department of Aeronautical Engineering, Indian Institute of Science in 197 where he is currently employed as a Scientific Officer. His fields of interest are solid state microwave circuits and antenna theory.

S. Ramakrishna, for a photograph and biography please see page 330 of the May 1982 issue of this TRANSACTIONS. 